\title{
TRIZ-based Patent Design Around for Enterprises: A Pencil Extender Device Innovative Redesign
}

\author{
Hai-yan Zhang ${ }^{1}$, Si-yuan Cheng ${ }^{1,2^{*}}$, Xue-rong Yang $^{1}$, Jing-ping Zhou ${ }^{1,2}$, Rui Wang ${ }^{1}$ \\ ${ }^{1}$ School of Electromechanical Engineering, Guangdong University of Technology, \\ Guangzhou, CHINA \\ ${ }^{2}$ Key Laboratory of Innovation Method and Decision Management System of Guangdong Province, \\ Guangzhou, CHINA
}

\begin{abstract}
Patent design around is crucial to the enterprise's product design and technology innovation. The patent-around innovative design method based on TRIZ is proposed, using function analysis to analyze the existed patent, providing the process and method for the enterprise's in-depth analysis of patent documents by standing in the patent-around perspective. Combining the around strategies and TRIZ problem-solving tools, it finds out the relationship between them, thus provides guidance for enterprises' technical personnel and designers to utilize suitable TRIZ problem-solving tools to solve the problem that arose after using the patent around strategies, to design a new product which is different to the existed patent. The proposed method may help small and medium enterprises, especially those who are not familiar with TRIZ theory, to utilize TRIZ theory effectively for patent-around and product innovation design. Finally, the method has been tested and verified successfully by a case of a pencil extender device. In this case, the patent around strategy of deletion and substitution method is used, then corresponding TRIZ
\end{abstract} problem-solving tools, contradiction matrix and knowledge-effect database are applied to solve the problems. Thus a new design around product which is more convenient is gotten.

Keywords-TRIZ; patent design around; pencil extender device; innovative design

\section{INTRODUCTION}

As the intensification of economic globalization and market competition, enterprises' demand for product innovation is becoming more and more important. There are many ways for product to realize innovation design, but they all need the support of the existed knowledge and experience. As the patented knowledge has the characteristics of advance and timeliness, it is the most effective carrier of the technical information and has great realistic significance for enterprise's innovation ${ }^{[1]}$.However, the enterprise often encounter patent barriers when they take advantage of patents, so they should have the awareness of patent strategy.

Patent-around design is one of the most important strategies of patent strategy. Initially, it is the America's legitimate competitive behavior and only used as a passive protective measure when their products are accused infringement ${ }^{[2,3]}$. But now patent design around is a kind of active defense and offensive strategy, and is helpful for enterprises doing invention activities. TRIZ is the Russian acronym for Theory of Solving Inventive Problems, was founded in 1946 by a Russian engineer and scientist, Genrich Altshuller ${ }^{[4]}$. TRIZ is an effective method to analysis technical system and solve technology problems met in invention activities.

At present, some researchers have proposed several approaches for TRIZ-based patent design around ${ }^{[5,6,7,8]}$. Many of them have given the general process of patent design around, which may guide the technical personnel to carry out patent design around to some degree. However, they did not give a detailed description for the application of TRIZ, for instance, how to apply the function analysis method to the patent analysis, or how to utilize TRIZ problem-solving tools after choosing around strategies, which may hinder the enterprises (especially for those small and medium enterprises who are not familiar with TRIZ theory) to design around.

Based on the research of others, TRIZ 
Function-analysis to patent analysis is proposed in this paper, which reveals the relationship between patent around strategies and TRIZ problem-solving tools, and could help enterprises and designers doing product innovative design more easily and effectively.

\section{PATENT DESIGN AROUND METHOD BASED ON TRIZ}

\section{A. TRIZ Theory}

Based on the study of the worldwide high level invention patents, TRIZ theory is a complete theoretical system of innovative method. Since TRIZ was generalized from the patent, it is also an effective tool of patent analyzing and provides a systematically and revolutionary new way for problem-solving. The theoretical framework of TRIZ is illustrated in figure $1^{[9]}$.As shown in Fig.1, TRIZ is a powerful theory for problem formulating, system analysis, contradiction solving, etc, and a tool which can help technical personal of enterprises improve their efficiency of innovation significantly.

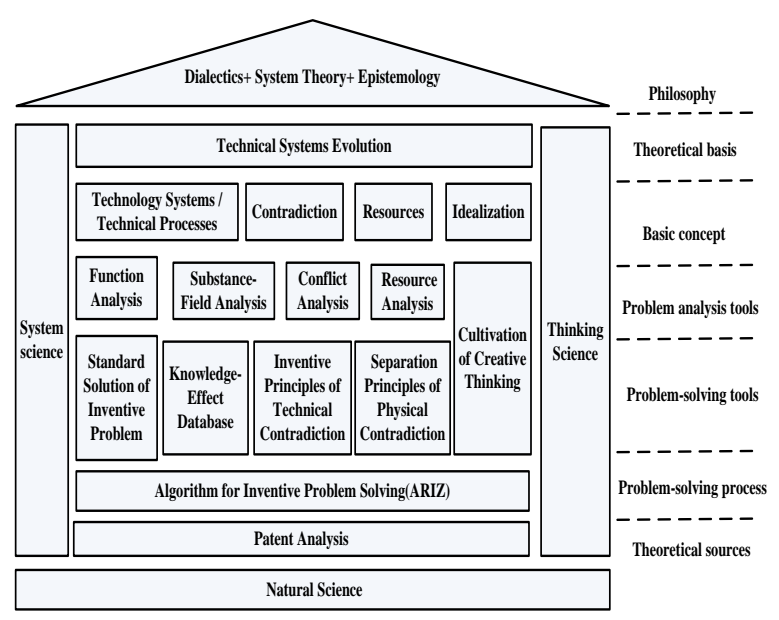

Fig. 1. Theoretical framework of TRIZ.

In this paper, the main tools used for patent-around design are the function analysis and the problem-solving tools. The function analysis is used to analyze the existed patent, and then the problem-solving tools are combined with patent around strategies for solving the innovation problems and designing a new product which is different to the existed patent.

\section{B. Methods of TRIZ-based Patent-around innovative design}

Generally, the process of TRIZ-based patent-around innovative design is mainly composed of the following four steps ${ }^{[10]}$ :

- Step1: Searching and analyzing the related patents to excavate the core technology of the studied product and identifying the target patent.

- Step2: Using function analysis to analyze the target patent. Get the direction of patent-around according to the function model.

- $\quad$ Step3: Using patent-around strategies and TRIZ problem-solving tools to find the innovative breakthrough and forming the design concept.

- Step4: Refining and optimizing the design concept to develop a new product and utilizing patent infringement for a test.

In this paper, we will focus on the depth analysis of the target patent by using of function analysis, and will build a function model to clear the advantages and disadvantages of target patent. Moreover, for the target patent which has been analyzed, this paper proposes some suggestions for using TRIZ innovative problem-solving tools after adopted patent around strategies so that the technical personnel can facilitate innovation more quickly and efficiently. And this is the main contribution of this study to others.

1) Patent analysis though the function analysis

Before patent analysis, enterprises should orientate the market and formulate patent searching strategy. The patent searching should focus on the inventive and new practicable patents for these patents implying the core technologies. Through a specific analysis of searched patents, one or several core patents are identified as the target patent.

The next step is analyzing the target patent and establishing a function model. Function analysis is an important tool of TRIZ for system analyzing, and its main purpose is to transform the abstract system into a figure for the designer to understand the whole technology system and its problems. The function model could help designers simplifying a complicated system and doing innovative design reasonable. Prior to this, the "interaction matrix" over the system and super system components shown in Table.1 can be established to identify and describe the interaction between system and super system components, thus to help designers create the function model of the target patent. 
TABLE I

COMPONENT INTERACTION MATRIX.

\begin{tabular}{|c|c|c|c|c|}
\hline & Component 1 & Component 2 & ....... & Component $\mathrm{n}$ \\
\hline Component 1 & & - & + & - \\
\hline Component 2 & & & - & - \\
\hline ....... & & & & + \\
\hline Component $\mathrm{n}$ & & & & \\
\hline
\end{tabular}

"+" indicates there is a relationship between two components

"-" indicates there is no relationship between two components

In establishing function model of the target patent, we must also analyze the patent documents, especially the independent claims and implementation methods, which will help us to identify the key components that may be avoided. Then, combine this and the function model to identify the direction of patent-around. The patent analysis process based on functional analysis of TRIZ is shown in Fig.2.

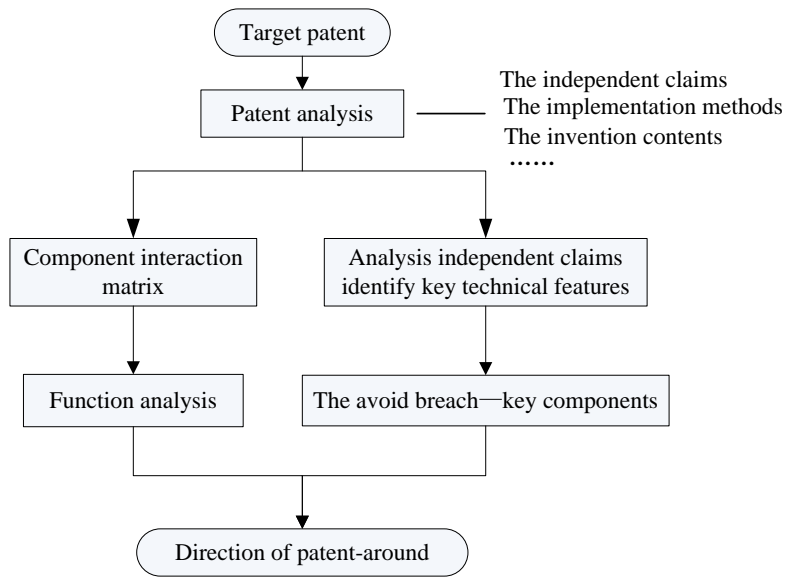

Fig. 2. The patent analysis process based on functional analysis of TRIZ.

2) Combination of design around strategies and TRIZ problem solving tools

After established the function model of the target patent, the next step is to use around strategies and TRIZ problem-solving tools for designing around and design a new product.

From Fig.1, the tools used in problem-solving phase include Standard Solutions of Inventive Problem, Knowledge-Effect database, Inventive Principles of Technical Contradiction and Separation Methods of Physical Contradiction. The standard Solutions mean that very similar problem model of different problems have the same solution model. It is used in a problem which consists of two or more unwanted interaction between subsystems, and providing solutions for those missing, inadequate, excess or harmful situations. The Knowledge-Effect database is a problem-solving tool which is based on the knowledge, and its application is by using the relevant knowledge of this field or other field to solve problems in design process. Inventive Principles represent a set of good ideas ready to be used, they are suitable for the technical conflict problems which are easily described by 39 engineering parameters. The application is not particularly complex and usually it comes in combination with the Contradiction Matrix. Separation principles are suitable for those physical conflicts which hide more deeply and intensely, the core idea is to achieve the separate of the contradiction between two sides.

At present, the commonly patent around strategies mentioned in the literature are the deletion, substitution, combination and decomposition method and exclusion feature substitution method. The exclusion feature substitution method involves search history of patent application and follow-up procedures, which is not related with innovative design, so here we will not discuss it.

After around strategies are applied to the functional model, usually there will get some new problems. To solve these problems, technicians generally seek TRIZ tools which are mentioned above to solve the problem, the process is complex and aimless. Here, the paper combine the around strategies and problem-solving tools, give the corresponding optimal problem-solving tools for each around strategies to provide some guidance and advice for enterprises to solve the problem.

Deletion means to reduce the number of elements in the claims to satisfy the all elements rule. In this way, contradiction or function loss is often appeared, then the problem could be converted to a technical or physical contradiction. At this time, choose Invention Principles to solve technical contradictions and the Separation Principles, Substance-Field Model and Standard Solutions, Knowledge-Effect Database to solve the physical contradictions.

Substitution means to replace some components with other components, by using different methods (technology, method, principle) to achieve the same function and effect 
of the original system and to satisfy the all elements rule and the doctrine of equivalents. If adopted this strategy, use the Knowledge-Effect Database to search other areas and find solutions.

Combination and decomposition refers to the combination of one or more technical features of system, or use a number of new features work together instead of one feature of the original patent claims to achieve the required functions to satisfy the all elements rule and the doctrine of equivalents. Knowledge-Effect Database may be considered a good tool of finding resources and solutions in this way.

The results of the analysis described above are summarized in table 2.

TABLE II THE COMBINATION OF PATENT AROUND STRATEGIES AND TRIZ PROBLEM-SOLVING TOOLS

\begin{tabular}{cccc}
\hline $\begin{array}{c}\text { Patent around } \\
\text { strategies }\end{array}$ & $\begin{array}{c}\text { The graphical expression } \\
\text { Of around strategies }\end{array}$ & $\begin{array}{c}\text { The avoided patent } \\
\text { infringement }\end{array}$ & $\begin{array}{c}\text { Recommended TRIZ } \\
\text { problem-solving tools }\end{array}$ \\
\hline Deletion & $\mathbf{A}+\mathbf{B}+\mathbf{C}+\mathbf{D} \rightarrow \mathbf{A}+\mathbf{B}+\mathbf{C}_{1}$ & the all elements rule & $\begin{array}{c}\text { Invention Principles } \\
\text { Separation Principles } \\
\text { Substance-Field Model } \\
\text { Standard Solutions } \\
\text { Knowledge-Effect Database }\end{array}$ \\
Substitution & $\mathbf{A}+\mathbf{B}+\mathbf{C}+\mathbf{D} \rightarrow \mathbf{A}+\mathbf{B}+\mathbf{C}_{1}+\mathbf{D}_{1}$ & $\begin{array}{c}\text { the all elements rule } \\
\text { the doctrine of equivalents }\end{array}$ & $\begin{array}{c}\text { Knowledge-Effect } \\
\text { Database }\end{array}$ \\
$\begin{array}{c}\text { Combination and } \\
\text { decomposition }\end{array}$ & $\begin{array}{c}\mathbf{A}+\mathbf{B}+\mathbf{C}+\mathbf{D} \rightarrow \mathbf{A}+\mathbf{B}+\mathbf{E} \\
\mathbf{A}+\mathbf{B}+\mathbf{C}+\mathbf{D} \rightarrow \mathbf{A}+\mathbf{B}+\mathbf{C}+\mathbf{D}_{1}+\mathbf{D}_{2}\end{array}$ & $\begin{array}{c}\text { the all elements rule } \\
\text { the doctrine of equivalents }\end{array}$ & $\begin{array}{c}\text { Knowledge-Effect } \\
\text { Database }\end{array}$ \\
\hline
\end{tabular}

The conclusion given above is just a suggestion, we can also look for other tools when using the recommended TRIZ tools can not solve the problem. After getting the conceptual scheme of the around design, the next step is to refine and complete the whole design of the new avoided product. Of course, patent infringement check is needed both in the design process and after the design is completed.

\section{A CASE EXAMPLE-THE CIRCUMVENTION OF A PENCIL EXTENDER DEVICE PATENT}

The pencil is a kind of tools for writing and drawing, its biggest advantage is that the correction can be easily and repeatedly erased, but also it has the disadvantages of fast using. In particular, each pencil always remain a section of pencil head, which are all thrown out because of the inconvenient of using, and thus resulting a waste of resources. Therefore, the invention of the pencil assist device has become the needs of today's society. Then we will use the method proposed above to design around.
Firstly, we should search and analysis the related patents to excavate the target patent of the pencil assist device. Through the keyword and IPC searching, as well as reading and analyzing of the contents of each patent, ultimately we determine "a pencil extender device" (CN $102381083^{[11]}$ )as the target patent.

Then, analyzing the target patent by using the process proposed above. After reading and analyzing specific content of the patent, we find that there are five key components, which also are the key technical features of this patent. They are 1-extension pipe, 2-screwed sleeve, 3-pushing rod, 4-convex ring and 5-clamping piece respectively, as shown in Fig. 3.

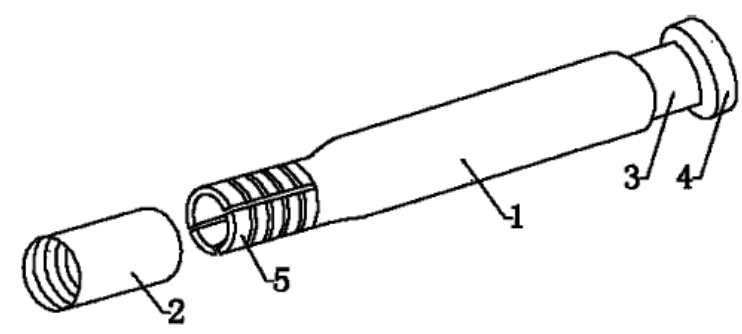

Fig. 3. The structure of the pencil extender device

Analysis the patent over all system components and super-system components, and the relationships between each other of them, then establish the components interaction matrix, shown in Table 3.

TABLE III THE COMPONENTS INTERACTION MATRIX OF THE PENCIL EXTENDER DEVICE

\begin{tabular}{ccccccc}
\hline & pencil & $\begin{array}{c}\text { extension } \\
\text { pipe }\end{array}$ & $\begin{array}{c}\text { screwed } \\
\text { sleeve }\end{array}$ & $\begin{array}{c}\text { pushing } \\
\text { rod }\end{array}$ & $\begin{array}{c}\text { convex } \\
\text { ring }\end{array}$ & $\begin{array}{c}\text { clamping } \\
\text { piece }\end{array}$ \\
\hline $\begin{array}{c}\text { Pencil } \\
\text { extension pipe }\end{array}$ & - & - & - & + (push) & - & + (clamp) \\
screwred sleeve & - & - & - & + & - & + \\
push rod & + & + & - & - & - & + (press) \\
convex ring & - & - & - & + & + (connect) & - \\
clamping piece & + & + & + (abrasion) & - & - & - \\
\hline
\end{tabular}

From the matrix, we see that the extension pipe have interactions with other components, but the relationship is not related with the system problem, so we'll not consider the extension pipe at the following process. Finally, a function model of this target patent has been established based on this matrix, which is shown in Fig. 4. From the function model, we find out the direction of design around: a. Improve the effects of excessive pushing; b. Eliminate the harmful effects of abrasion. 


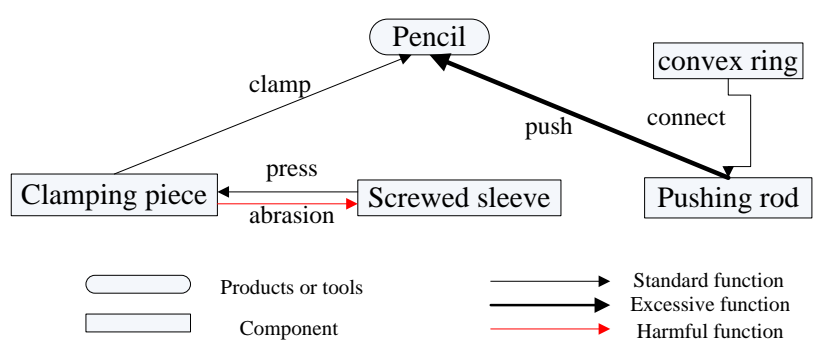

Fig.4. The function model of the pencil extender device

Thirdly, utilizing the around strategies and TRIZ problem-solving tools to improve system performance of the pencil extender device. Consider using the around strategies to solve the problem above, a new function model has gotten as shown in Fig. 5.

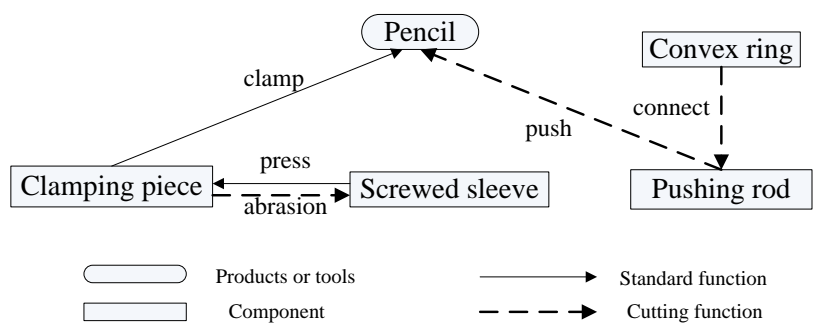

Fig. 5. The new function model of the pencil extender device

For problem a, we choose deletion method and try to delete the excessive effect of "push". According to the table.2, we translate this problem into standard TRIZ problem and use technical conflicts solving method to solve this problem. The improved parameter can be identified as 33-easy of operation, the deteriorated parameter is 32-easy of manufacture. By looking up the contradiction matrix, we find the invention principles are NO.2 Taking out, NO.5 Merging and NO.12 Equipotentiality. The NO.2 Taking out means to extract any features of the product, so as to reduce the complexity and does not affect the whole function mechanism. According to the inspiration of the principle NO.2, delete pushing rod and convex ring of the original patent, and the exposed pencil length can be adjusted directly by loosening screw sleeve, so there is no need to have such function of pushing carried by pushing rod and convex ring.

For problem b, choose substitution method to find out another structure that can substitute the screw-thread fit between screwed sleeve and clamping piece. According to table.2, use the Knowledge-Effect database to solve this problem. We search the database, and find out that the structure of square saw-tooth is better than screw-thread fit, which can overcome the wear and tear resulted from screw-thread fit.

The last step is to refine and optimize the design concept above and do infringement judgment for the new product. Finally, a totally different new product has gotten. It mainly includes two structures, which are extension pipe and tighten set. The tighten set is placed in the middle part of the extension pipe, which can be moved up and down to clamp the clamping piece. With this structure, the loss of tighten set which may caused by user's careless can be avoided. Delete pushing rod, pencil can be pushed directly from the clamping piece. Furthermore, adopt the square saw-tooth mating between tighten set and clamping piece, which can effectively improve the convenience of the system and extend the life of the product. Finally, a new product that avoided the original patent has came out, as shown in Fig. 6.

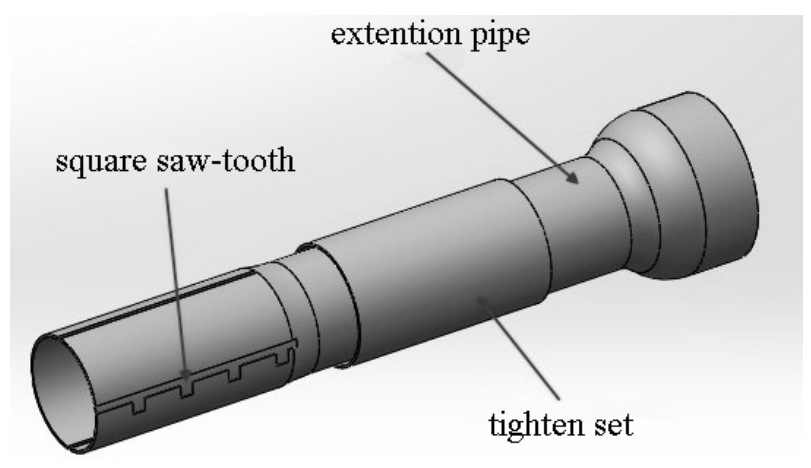

Fig. 6. The new product of the pencil extender device

\section{CONCLUSION AND DISCUSSION}

The paper combined the function analysis and TRIZ problem-solving tools with patent design around to develop a new product. The function analysis could help designers understand the target patent more deeply, dig out core technologies and shortcomings of the target patent. Meanwhile, gathering around strategies and TIRZ problem-solving tools together could help technicians for choosing innovative approaches more effectively, and promote application of TRIZ in the enterprises. A pencil extender device is carried out in this paper to verify the method, and a new design around product is gotten. This method has practical significance and guidance for enterprises and designers. 


\section{ACKNOWLEDGEMENTS}

This research was financially supported by the Technology Projects of Guangdong Province, China(No.2013B061000006,2014A040402004,2014A040 401078).

\section{REFERENCES}

[1] LIU Zhen-tao,“ Research on proprietary knowledge services and ITS platform for small and medium-sized enterprises,” D. in press .Shang hai, Shanghai Jiao Tong University,2007.

[2] J.Thomas McCarthy, "McCarthy's desk encyclopedia of intellectual property,” The Bureau of National Affairs, Inc;1991.

[3] Liu, Y., Jiang, P., Wang, W., \& Tan, R. , “ Integrating requirements analysis and design around strategy for designing around patents,"IEEE International Conference on Computing,Control and Industrial Engineering, Vol.2, pp.29-32,2011.

[4] Terninko, J, Zusman, A, and Zlotin, B, "Systematic innovation-An introduction to TRIZ,” New York: St.Lucie Press, 1998.
[5] LI Peng, AN Ji-ping, "Discussion on application of TRIZ theory in patent around design ,’J. in press .CHINA Invention \& Patent, Vol.2, pp.29-32,2013.

[6] Jiang, P., Zhai, J., Chen, Z., and Tan, R., "The patent design around method based on TRIZ," IEEE International Conference on Industrial Engineering and Engineering Management pp.1067-1071,2009.

[7] SHI Bing-xuan, "Research on strategy of design around patent,” D. in press . HangZhou , Zhejiang University ,2006.

[8] ZHANG Xiang-tang, ZHOU Wei-cheng, “ Process of the design around the patent group based on correlation analysis of components,” C/CD. in press. Taizhong, China: The Eleventh Symposiumon Taiwan, 2006.

[9] SHI Rongming, ZHAO Min, SUN Cong, "Knowledge engineering and Innovation,” BeiJing: Aviation Industry Press, China,2011.

[10] JIANG Ping, LUO Ping-ya, "Method about patent design around based on function trimming," J. in press. Journal of Mechanical Engineering, Vol. 48,pp. 46-54,2012.

[11] GAO Hong wei, “A pencil lengthening device,” P. in press. China, 201010265651.5, March 2012. 